\title{
Cracking the immunological code of pregnancy
}

Interactions between maternal immune cells and the placenta influence pregnancy complications

\section{ByFrancesco Colucci}

The World Health Organization estimates that $10 \%$ of the burden of disease worldwide is due to problems that arise during pregnancy which compromise the health of mother and fetus. Genetic, endocrine, metabolic, and cardiovascular factors contribute to pregnancy complications, e.g spontaneous abortion, pre-eclampsia, fetal growth restriction, preterm labor and still birth. Some of these problems are due to placental dysfunction ( 7 . Attaching to and invading deep into the specialized uterine mucosa called decidua, which is rich in maternal immune cells, the placenta is the fetus's lifeline. It grows from the blastocyst-derived trophoblast and, once formed, nourishes the fetus trough the umbilical cord. Carrying genetic material from another individual (the father), the placenta ought to be targeted by the immune system that specializes in detecting and destroying what is different from self. Significant pathology, including allergy, autoimmunity, transplant rejection, and sepsis is caused by exaggerated, inappropriate, unwanted, or systemic immune responses. Do interactions between the placenta and maternal immune system influence pregnancy complications? And if we understand these interactions, can we intervene to improve pregnancy outcome?

One well-documented example of how the maternal immune system can cause pathology through immunological incompatibility is Rhesus (Rh) disease. Immunity is passed on from mother to fetus through special receptors on placental cells that bind antibodies of a specific type (IgG), which are best at carrying immunological memory. This arms neonates with their mother's antibodies, which protect infants while they develop their own antibodies but, in pregnant Rh- mothers bearing a Rh+ positive fetus, harmful maternal antibodies against incompatible blood groups are also transported through the placenta and destroy fetal red blood cells causing severe anemia. Understanding the basic mechanisms of this fetal disease has led to a simple and successful preventative strategy based on immune intervention.

To understand most pregnancy complications, however, one ought to take a closer look at the interactions within the decidua (Figure). Trophoblast invasion into the decidua is a necessary event to ensure that the developing embryo taps into nutrients and oxygen available in the maternal blood. The concerted effort between maternal decidual cells and fetal trophoblast cells transforms uterine arteries into flaccid, large conduits, called spiral arteries, where circulation is slowed down. Appendices of the placenta (the villi) bathe in the transformed arteries, both absorbing the necessary nutrients and exchanging gases needed for fetal growth. It is when placental invasion is too shallow and arterial transformation incomplete that some pregnancy complications arise (1). Progress in obstetrics research is hard because of the constantly changing nature of tissues and the difficulty in accessing them. Naturally, the placenta is available at term, but key events happen in the first trimester. An additional challenge in modern immunology is to decode the function of tissue resident immune cells, which despite phenotypic resemblance across organs, may adapt in response to specific microenvironments. Moreover, each species has evolved its own way of placentation. Bearing in mind the differences in anatomy and reproductive biology, the mouse may be a useful model. The placenta in both species is haemochorial, meaning extravillous trophoblast (EVT) cells invade into the decidua in both species (Figure), although the degree of invasion is greater in humans. Another similarity between human and mouse is that cells of the innate immune system are most abundant in the decidua. These include decidual NK cells (dNK) and macrophages, along with dendritic cells (DC) and T cells, including regulatory $T$ cells (Tregs) (2). Mouse studies have helped to appreciate the critical role played in pregnancy by Tregs, a special population of lymphocytes that suppress immune responses. Tregs are induced in the female reproductive tract by cytokines in the seminal plasma, prevent fetal loss, expand in subsequent pregnancies, and, in humans, may be at fault in certain pregnancy complications (3). 
And yet, antigen-specific maternal immune cells that might cause placental dysfunction and must be controlled by Tregs, have never been identified. It is possible that the physiological role of decidual Tregs is not actually to suppress immune responses. An alternative role for decidual Tregs may be tissue homeostasis, as already shown in adipose tissue, muscle, and intestine (4).

Maternal immune cells in the decidua may have adapted to have a physiological function rather than specializing in aggressive immunological defense. Immunological mechanisms originally evolved to destroy and repair, might have been co-opted to participate to successful placental invasion. Inflammation, intended as the stereotypical vascular and cellular adaptations that respond to tissue injury by destroying first and repairing after, may well be an intrinsic feature of reproduction, rather than being an obstacle. Clearly there are extreme cases when local inflammation caused by infections or systemic endothelial responses in pre-eclampsia are unhealthy and can be even fatal to mother or fetus. But, operating constantly and independently of conception and implantation, inflammation and the innate immune system might have become integral to the necessary destruction and regeneration of the uterine mucosa during the menstrual cycle. It is therefore conceivable that the process of placental invasion and differentiation have also benefited from the function of the immune system. The concept that maternal immune cells must be activated for a healthy pregnancy may be counterintuitive to traditional concepts of immunity and pregnancy, which suggest that immunological tolerance must prevail and, for the pregnancy to succeed, maternal immune cells must be prevented from responding to paternal antigens.

The ultimate example of the need for functional immune cells at the maternal-fetal interface may well be dNK cells. While blood NK cells are known for their natural propensity to kill virally-infected and cancerous cells, dNK are involved in tissue physiology, rather than in killing. Original observations in mice demonstrated indeed that dNK cells are necessary for uterine vascular adaptations during pregnancy (5). In both human and mice dNK cells are found in close proximity to both trophoblast at sites of invasion and blood vessels. It is reasonable to think that dNK cells in both species participate in controlling trophoblast invasion and transforming the uterine blood vessels. Evidence suggests that excessive inhibition of dNK cell function impedes full development of the placenta with repercussions on fetal growth (6). Maternal dNK cells and trophoblast cells can be seen establishing intimate connections. Several receptor-ligand interactions have been decoded that can mediate the dialogue between mother and placenta (Figure). Importantly, the main determinants of histocompatibility and graft rejection (the extremely variable HLA molecules) are excluded from this dialogue because the outer layer of the placenta (syncytiotrophoblast) does not express them, thus avoiding any possible recognition by maternal immune cells. EVT however do express polymorphic HLA-C. All HLA-C allotypes can bind polymorphic $\mathrm{dNK}$ receptors called $\mathrm{KIR}$, which can be activating or inhibitory. Certain combinations of maternal $K I R$ and fetal $H L A-C$ genetic variants are indeed linked to birth weight and pregnancy complications (6). Modulation of dNK cells activation by this interaction may sway the balance towards too much inhibition or activation. Similar mechanisms seem to operate in mice, where dNK cells are key to promoting fetal growth ( $\nearrow$. Other receptor-ligand pairs between dNK cells and trophoblast may be key to placentation, such as receptors LILRB1 (also expressed on macrophages) and NKG2A, which bind respectively to HLA-G and HLA-E. Memory granting us adaptive immunity upon infection or vaccination has been thought for decades to be a feature of $B$ and $T$ lymphocytes. It has now become apparent, however, that innate immunity also has some form of memory. During first-time pregnancies, LILRB1 and NKG2C (also binding HLA-E) may trigger epigenetic DNA modifications in some dNK cells, making them potentially more active in subsequent pregnancies (8), which are exposed to reduced risk of pre-eclampsia.

The cellular networks operating in the decidua and placenta are starting to be revealed by new technology. RNA sequencing in dNK and related innate lymphoid cells in mice has revealed further specialization of these cells in the decidua, with potential division of labor and adaptation to the different phases of reproductive life (9). Single-cell RNA sequencing of the human maternal-fetal 
interface has begun to identify cellular and molecular networks that involve decidual stromal cells, immune cells, epithelial and endothelial cells, as well as fetal trophoblast and macrophages (10). The three subsets of dNK cells identified may well have distinct functions connecting with trophoblast, maternal stromal or immune cells (19). Mass cytometry, both on cell suspensions and by imaging may well reveal new functional and spatial information on these interactions, because it lends itself to a detailed analysis of heterogeneous cell populations. Human trophoblast organoids will help to study complex interactions with other cells at the maternal-fetal interface, including immune cells, and in defined genetic backgrounds (11).

Maternal and fetal cells in the decidua and placenta don't operate in isolation. How do nutrition, metabolism, and oxygen concentration influence these interactions? Metabolomics analyses of the placenta have started to decipher the differences between metabolites in maternal and fetal tissues (12) and might eventually integrate our growing appreciation of how metabolism shapes immune functions. Do microbes influence immunology of placentation, contributing to pregnancy complications? Labor may indeed be precipitated by infections leading to pre-term birth. The history of ancestral viral infections that have helped placentation is written in our DNA. Our genomes harbor endogenous retroviruses that have helped placental cells to fuse together to form a syncytium. Microbes or microbial products living in the mother may influence feto-placental development, and some evidence suggests that microbiota in the placenta and amniotic fluid may colonize fetal gut in utero (13). However, other findings do not support the existence of a placenta microbiome (14).

Once cellular and molecular networks and the factors that regulate them are identified the next challenge will be to understand how they go wrong in pregnancy complications. Are we ready to manipulate the human immune system during pregnancy to prevent complications? The short answer is not yet. Despite the successful precedent of immune intervention to prevent Rhesus disease for over 5 decades, one may argue that it may be too risky to interfere with the maternal immune system. Currently, anti-inflammatory and immunosuppressive drugs such as aspirin, hydroxycloroquine, and steroids are used to improve the outcome of some pregnancy complications like pre-eclampsia, antiphospholipid antibodies, chronic villitis or even to improve fertility. The benefit of these treatments is generally minimal and, for example in pre-eclampsia, may be confounded by the beneficial effect of aspirin on the systemic endothelial response. In modern medicine there is much excitement about the power of immune checkpoint inhibitors $(\mathrm{ICl})$ in the treatment of patients with cancer. These monoclonal antibodies target inhibitory receptors on lymphocytes and, in a significant number of patients, reawake lymphocytes against cancer, improving outcome. Subsets of dNK cells and T cells in the decidua have inhibitory receptors that can be targeted by ICI (NKG2A, KIR, TIGIT, TIM-3 on dNK cells and CTLA-4 and PD-1 on T cells) and trophoblast cells express ligands for some of these receptors, including HLA-E, HLA-C, PVR, and PD-L1 (19). ICI in pregnant mice causes fetal loss. However, a patient with melanoma treated with $\mathrm{ICl}$ survived and had a favorable pregnancy outcome (15), illustrating that the possibility of immune-based interventions at the maternal-fetal interface may be explored. With technology advancing fast and by improving our knowledge of both the individual uterine immune cells and the function of the whole system, we are starting to crack the unique immunological code of pregnancy and may one day intervene to improve outcome.

\section{/1885 words - 15 references}




\section{Figure legend}

The placenta develops during the first trimester, when trophoblast cells replace the lining of maternal arteries, which are transformed into spiral arteries. The syncytiotrophoblast (Syn) that is in direct contact with maternal blood does not express MHC molecules and is therefore not recognized by maternal immune cells. Fetal extravillous trophoblast (EVT) bud off the villi and interact with maternal immune cells. Some of the molecular interactions between macrophages, dNK cells, dendritic cells and fetal EVT are indicated. Decoding the nature of these interactions and how they affect placental development my help to understand the cause of some pregnancy complications. Modified from Robbins JR, Skrzypczynska KM, Zeldovich VB, Kapidzic M, Bakardjiev Al (2010) Placental Syncytiotrophoblast Constitutes a Major Barrier to Vertical Transmission of Listeria monocytogenes. PLoS Pathog 6(1): e1000732. https://doi.org/10.1371/journal.ppat.1000732. 


\section{References}

1. I. Brosens, R. Pijnenborg, L. Vercruysse, R. Romero, The "Great Obstetrical Syndromes" are associated with disorders of deep placentation. Am J Obstet Gyneco/204, 193-201 (2011).

2. A. Erlebacher, Immunology of the maternal-fetal interface. Annu Rev Immuno/31, 387-411 (2013).

3. S. A. Robertson et al., Therapeutic Potential of Regulatory T Cells in Preeclampsia-Opportunities and Challenges. Front Immuno/ 10, 478 (2019).

4. M. Panduro, C. Benoist, D. Mathis, Tissue Tregs. Annu Rev Immuno/34, 609-633 (2016).

5. A. A. Ashkar, J. P. Di Santo, B. A. Croy, Interferon gamma contributes to initiation of uterine vascular modification, decidual integrity, and uterine natural killer cell maturation during normal murine pregnancy. The Journal of experimental medicine 192, 259-270 (2000).

6. A. Moffett, F. Colucci, Uterine NK cells: active regulators at the maternal-fetal interface. J Clin Invest 124, 1872-1879 (2014).

7. J. Kieckbusch, L. M. Gaynor, A. Moffett, F. Colucci, MHC-dependent inhibition of uterine NK cells impedes fetal growth and decidual vascular remodelling. Nat Commun 5, 3359 (2014).

8. M. Gamliel et al., Trained Memory of Human Uterine NK Cells Enhances Their Function in Subsequent Pregnancies. Immunity 48, 951-962 e955 (2018).

9. I. Filipovic et al., Molecular definition of group 1 innate lymphoid cells in the mouse uterus. Nat Commun 9, 4492 (2018).

10. R. Vento-Tormo et al., Single-cell reconstruction of the early maternal-fetal interface in humans. Nature 563, 347-353 (2018).

11. M. Y. Turco et al., Trophoblast organoids as a model for maternal-fetal interactions during human placentation. Nature 564, 263-267 (2018).

12. J. M. Walejko, A. Chelliah, M. Keller-Wood, A. Gregg, A. S. Edison, Global Metabolomics of the Placenta Reveals Distinct Metabolic Profiles between Maternal and Fetal Placental Tissues Following Delivery in Non-Labored Women. Metabolites 8, (2018).

13. M. C. Collado, S. Rautava, J. Aakko, E. Isolauri, S. Salminen, Human gut colonisation may be initiated in utero by distinct microbial communities in the placenta and amniotic fluid. Sci Rep 6 , 23129 (2016).

14. J. S. Leiby et al., Lack of detection of a human placenta microbiome in samples from preterm and term deliveries. Microbiome 6, 196 (2018).

15. M. Burotto et al., Viable Pregnancy in a patient with metastatic melanoma treated with double checkpoint immunotherapy. Semin Onco/45, 164-169 (2018). 